\title{
Quantum Geometry and Black Hole Entropy
}

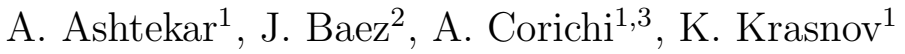 \\ 1. Center for Gravitational Physics and Geometry, \\ Pennsylvania State University, PA 16802, USA \\ 2. Department of Mathematics, University of California, \\ Riverside, CA 92521, USA \\ 3. Instituto de Ciencias Nucleares, UNAM \\ A. Postal 70-543, México D.F. 04510, México
}

\begin{abstract}
A 'black hole sector' of non-perturbative canonical quantum gravity is introduced. The quantum black hole degrees of freedom are shown to be described by a Chern-Simons field theory on the horizon. It is shown that the entropy of a large non-rotating black hole is proportional to its horizon area. The constant of proportionality depends upon the Immirzi parameter, which fixes the spectrum of the area operator in loop quantum gravity; an appropriate choice of this parameter gives the Bekenstein-Hawking formula $S=A / 4 \ell_{P}^{2}$. With the same choice of the Immirzi parameter, this result also holds for black holes carrying electric or dilatonic charge, which are not necessarily near extremal.
\end{abstract}

PACS: 04.60.-m, 04.70.Dy 
The statistical mechanical origin of black hole entropy has drawn a great deal of attention recently (for reviews, see for example [1] [3]). Most of the work based on string theory has focused on the extremal and near extremal cases. The purpose of this letter is to introduce a new framework, based on non-perturbative quantum gravity [4. [5], that enables one to treat general black holes in four dimensions.

The basic ideas can be summarized as follows. We first introduce a sector of the classical theory that corresponds to isolated, non-rotating black holes and find the associated phase space description. Then we quantize the resulting phase space. Finally, we isolate the quantum states that describe the geometry of the horizon. It is these degrees of freedom that account for the black hole entropy in our approach. We find that the statistical mechanical entropy of the black hole is proportional to its horizon area.

Recently, non-perturbative techniques have led to a quantum theory of geometry in which operators corresponding to lengths, areas and volumes have discrete spectra. Of particular interest are the spin network states associated with graphs in 3-space with edges labelled by spins $j=\frac{1}{2}, 1, \ldots$ and vertices labelled by intertwining operators [6, [0]. If a single edge punctures a 2-surface transversely, it contributes an area proportional to $\sqrt{j(j+1)}$ [8, 9]. Over the last two years, this picture led to certain constructions which in turn inspired the present work. First, while working with a space-time region with boundary in Euclidean general relativity with non-zero cosmological constant, Smolin [10 was led to introduce gravitational surface states which could be identified with the states of the $\mathrm{SU}(2)$ ChernSimons theory on a surface with punctures. Second, Rovelli [11], motivated by the work of Krasnov [12], estimated the number of spin-network states which endow a 2-sphere with a given, large area and applied this estimate to black hole horizons. Third, Krasnov [13] proposed to combine the two sets of ideas by introducing certain boundary conditions on regions bounded by 2-spheres. Finally, Carlip's [国] considerations of surface states in the context of three dimensional black holes also played a suggestive role in the classical part of our treatment.

Let us begin with uncharged, non-rotating black holes. A sector of the classical phase space corresponding to an isolated, non-rotating black hole can be constructed as follows. Consider the manifold (with boundary) representing the asymptotic region of FIG. 1. We refer to the outer boundary as $\mathcal{I}$ and the inner boundary as $\mathcal{H}$. Our dynamical fields are a soldering form $\sigma_{a}^{A A^{\prime}}$ for $\mathrm{SL}(2, \mathrm{C})$ spinors and an $\mathrm{SL}(2, \mathrm{C})$ connection $A_{a A}{ }^{B}$ [四, 14]. (In a classical solution, $g_{a b}=\sigma_{a}^{A A^{\prime}} \sigma_{b A A^{\prime}}$ is the Lorentzian space-time metric and $A_{a A^{B}}{ }^{B}$ is the selfdual connection that operates only on unprimed spinors.) On $\mathcal{I}$, fields are required to satisfy the standard asymptotically flat boundary conditions. The conditions on $\mathcal{H}$, on the other hand, are more subtle and will be discussed in detail elsewhere. The key requirements are: i) $\mathcal{H}$ be a null surface with respect to the metric $g_{a b}$; ii) On a 'finite patch' $\Delta$ of $\mathcal{H}$, the area of any cross-section be a constant, $A_{S}$, the Weyl spinor be of Petrov type 2-2 and its only non-zero component, $\Psi_{2}$, be given by $\Psi_{2}=2 \pi / A_{S}$; and, iii) the 2-flats on $\Delta$, orthogonal to the two principal null directions of the Weyl tensor span 2-spheres and the pull-back of the connection $A_{a}$ to these 2 -spheres be real.

In what follows, we focus only on the part $\Delta$ of $\mathcal{H}$ and the corresponding region $\mathcal{M}$ of the spacetime (see FIG. 1). Roughly, condition ii) implies that there is no gravitational radiation falling into $\Delta$ (i.e., the black hole is 'isolated' there) while the first part of iii) implies that 
it is 'non-rotating'. The three conditions together imply that, on partial Cauchy surfaces (such as $M$ ) that intersect $\Delta$ in the preferred 2-spheres, the 2-spheres are marginally outer trapped surfaces. These boundary conditions have been extracted from the geometrical structure available at the Schwarzschild horizon. However, we do not require staticity and allow gravitational waves in the exterior region. Therefore, our phase space will be infinite dimensional; the boundary conditions are quite weak.

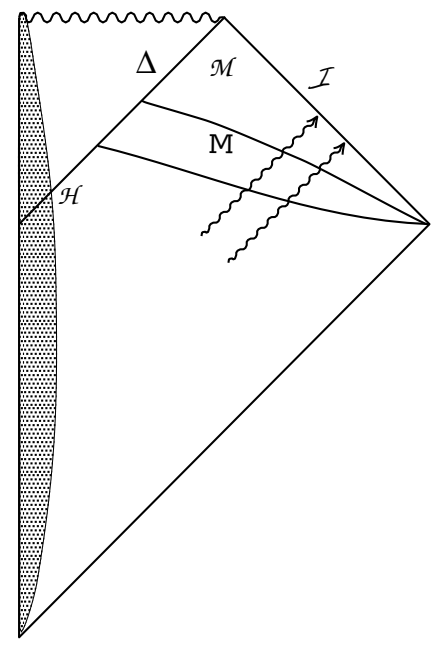

FIG. 1. Example of a spacetime of interest.

However, these boundary conditions are strong enough to imply that the variational principle is well defined. More precisely, we can add to the standard self-dual action [14] a surface term so that the total action is functionally differentiable and yields precisely Einstein equations:

$$
\begin{array}{r}
S(\sigma, A)=-\frac{i}{8 \pi G} \int_{\mathcal{M}} \operatorname{Tr}(\Sigma \wedge F) \\
-\frac{i}{8 \pi G} \frac{A_{S}}{4 \pi} \int_{\Delta} \operatorname{Tr}\left(A \wedge d A+\frac{2}{3} A \wedge A \wedge A\right) .
\end{array}
$$

Here $\Sigma_{a b}^{A B}=2 \sigma_{[a}{ }^{A A^{\prime}} \sigma_{b] A^{\prime}}{ }^{B}$ while $F_{a b A}{ }^{B}$ is the curvature of the connection $A$, and $G$ is Newton's constant. (Throughout we have set $c=\hbar=1$.) Note that the required surface term is precisely the action of Chern-Simons theory. It is straightforward to cast the theory into Hamiltonian form. The basic phase space variables are the restrictions of $\Sigma$ and $A$ to the spatial hypersurface $M$ with a boundary $S$. (Vector densities, dual to the pull-back of $\Sigma$, are the familiar density weighted triads.) Unfortunately, the restriction to $M$ of the self-dual connection $A$ is a complex valued $\mathrm{SU}(2)$ connection and the functional analysis required to handle complex connections in quantum theory is not yet fully developed.

Therefore, at this stage, it is easier to make a transformation to real variables [15. On $M, A$ can be expressed in terms of real fields as $A_{a}=\Gamma_{a}-i K_{a}$, where $\Gamma$ is the 3dimensional spin connection compatible with the triad field and $K$ is the extrinsic curvature of $M$. This suggests [16] that we introduce real phase space variables $\gamma_{a}:=\Gamma_{a}-\gamma K_{a}$ and $\Sigma_{a b}:=(1 / \gamma) \Sigma_{a b}$, where $\gamma$ is a positive real number known as Immirzi parameter. Then, our boundary conditions imply that the phase space consists of real fields $\left(\gamma_{a}, \Upsilon_{a b}\right)$ that are asymptotically flat at infinity and satisfy the following condition at $S$ : 


$$
\underline{F}_{a b}^{A B}=-\frac{2 \pi \gamma}{A_{S}} \underline{\gamma}_{a b}^{A B}
$$

where underbars denote pull-backs to $S$. This in turn implies that the restriction of $\gamma A_{a}$ to $S$ yields a reducible connection, i.e., one satisfying $D_{a} r=0$ for some 'radial' internal vector $r$. Although it is not necessary to do so, for technical simplicity, we fix $r$ on $S$ using the $\mathrm{SU}(2)$ gauge freedom. Then the gauge group on the boundary is reduced to $\mathrm{U}(1)$ and only the $r$ component of (2) is non-trivial.

On this manifestly real phase space, the symplectic structure derived from (1) is given by

$$
\begin{array}{r}
\left.\Omega\right|_{(\gamma A, \gamma \Sigma)}\left(\left(\delta^{\gamma} A, \delta^{\gamma} \Sigma\right),\left(\delta^{\gamma} A^{\prime}, \delta^{\gamma} \Sigma^{\prime}\right)\right)= \\
\frac{1}{8 \pi G} \int_{M} \operatorname{Tr}\left[\delta^{\gamma} \Sigma \wedge \delta^{\gamma} A^{\prime}-\delta^{\gamma} \Sigma^{\prime} \wedge \delta^{\gamma} A\right] \\
-\frac{k}{2 \pi} \oint_{S} \operatorname{Tr}\left[\delta^{\gamma} A \wedge \delta^{\gamma} A^{\prime}\right],
\end{array}
$$

where $\quad k=\frac{A_{S}}{8 \pi \gamma G}$

is later identified with the level of the Chern-Simons theory. Up to a numerical coefficient, $k$ is simply the area of the horizon of black hole measured in the units of Planck area $\ell_{P}^{2}=G$ [13. Note that, in addition to the familiar volume term, the symplectic structure has a surface term which coincides with the symplectic structure of the Chern-Simons theory.

The theory has three sets of first class constraints. A careful analysis shows that they generate the following gauge transformations: i) $\mathrm{SU}(2)$ internal rotations that reduce to $\mathrm{U}(1)$ rotations preserving a fixed vector $r$ on the boundary $S$; ii) spatial diffeomorphisms that leave $S$ invariant; and, iii) canonical transformations generated by the scalar constraint with lapse fields approaching zero at spatial infinity and on $S$. Somewhat surprisingly, it turns out that condition (2), the pull-back to $S$ of the type 2-2 requirement, ensures full gauge invariance on the boundary. Without it, as in the case of the scalar constraint, only the internal rotations whose generators vanish on $S$ could be regarded as gauge.

It is intuitively clear that not all the degrees of freedom described by fields $\gamma A, \Upsilon \Sigma$ are relevant to the problem of black hole entropy. In particular, there are 'volume' degrees of freedom in the theory corresponding to gravitational waves far away from $\Delta$ which should not be taken into account as genuine black hole degrees of freedom. The 'surface' degrees of freedom describing the geometry of the horizon $S$ have a different status. It has often been argued (see, e.g., [3] and references therein) that it is the degrees of freedom 'living on the horizon' that should account for the entropy. We adopt this viewpoint in our approach.

In the classical theory that we have described, the volume and surface degrees of freedom cannot be separated: all fields on $S$ are determined by fields in the interior of $M$ by continuity. However, in the quantum theory, the fields describing geometry become discontinuous in certain precise sense [6], and the fields on $S$ are no longer determined by fields in $M$; in this case there are independent degrees of freedom 'living' on the boundary. These surface degrees of freedom are the ones that account for black hole entropy in our approach. 
To quantize the theory, we first construct a Hilbert space $\mathcal{H}^{V}$ of 'volume' states and a Hilbert space $\mathcal{H}^{S}$ of 'surface' states, and then impose constraints on $\mathcal{H}^{V} \otimes \mathcal{H}^{S}$ to obtain the space of physical states. We take $\mathcal{H}^{V}$ to consist of certain square-integrable functions on the space of generalized $\mathrm{SU}(2)$ connections [6] on $M$ modulo gauge transformations that are the identity on $S$. The form of the Hilbert space $\mathcal{H}^{S}$ of surface states is motivated by the fact that in the quantum theory we wish to impose the boundary condition (2) as an operator equation. That is, given a spin network state $\Psi_{V}$ in $\mathcal{H}^{V}$ and a state $\Psi_{S}$ in $\mathcal{H}^{S}$, the quantum version of the $r$ component of equation (2) should read

$$
\left(1 \otimes \frac{2 \pi \gamma}{A_{S}} \underline{\hat{F}}_{a b} \cdot r+\underline{\hat{\Sigma}}_{a b} \cdot r \otimes 1\right) \Psi_{V} \otimes \Psi_{S}=0
$$

The structure of this equation implies that $\Psi_{V}$ and $\Psi_{B}$ should be eigenstates of $\underline{\underline{\Sigma}}_{a b} \cdot r$ and $\underline{\hat{F}}_{a b} \cdot r$ respectively. Now, the 'polymer nature' of quantum geometry in $M$ implies that eigenvalues of $\hat{\Sigma}_{a b} \cdot r$ are distributional, given by [9]

$$
8 \pi \ell_{P}^{2} \sum_{i} j_{i} \delta^{2}\left(x, p_{i}\right) \eta_{a b} \ell_{P}^{2}
$$

for some points $p_{i}$ on $S$, where $j_{i}$ are half-integers, $\delta^{2}$ is the delta distribution on $S, \eta_{a b}$ the Levi-Civita density on $S$ and $\ell_{P}$ the Planck length. Therefore, (5) implies that the surface states $\Psi_{S}$ have support only on generalized connections that are everywhere flat except at a finite number of points $p_{i}$. It turns out that such generalized connections can be identified with ordinary connections with distributional curvature. Since the surface symplectic structure is that of Chern-Simons theory, for any fixed choice

$$
\mathcal{P}=\left\{\left(p_{1}, j_{p_{1}}\right), \ldots,\left(p_{n}, j_{p_{n}}\right)\right\}
$$

of points in $S$ labelled by spins, we wish $\mathcal{H}^{S}$ to have a subspace given by the space of states of $\mathrm{U}(1)$ Chern-Simons theory on a sphere with punctures $p$ labelled by spins $j_{p}$. The total space $\mathcal{H}^{S}$ is the direct sum of these subspaces.

Note now that $(k / 2 \pi) \underline{\hat{F}}$ is the generator of internal rotations in Chern-Simons theory. Thus, the meaning of (5) turns out to be rather simple: it ensures that the volume and surface states are 'coupled' in precisely the correct way so that the total state is invariant under $\mathrm{U}(1)$ internal rotations at $S$. The remaining constraints require that the states be invariant under diffeomorphisms of $M$ that leave $S$ invariant and under motions generated by the Hamiltonian constraint smeared with any lapse field that vanishes at $S$. Thus, the following physical picture emerges. For each set $\mathcal{P}$ of finitely many punctures $p$ labelled by spins $j_{p}$, there is a subspace $H_{\mathcal{P}}^{V}$ of volume states having a basis given by open spin networks whose edges intersect $S$ only at these punctures, where they are labeled by the spins $j_{p}$. Similarly there is a subspace $H_{\mathcal{P}}^{S}$ consisting of quantum states of U(1) Chern-Simons theory on the punctured surface $S$. The total physical Hilbert space is given by:

$$
\mathcal{H}_{\text {phy }}=\frac{\oplus_{\mathcal{P}}\left[\mathcal{H}_{\mathcal{P}}^{V} \otimes \mathcal{H}_{\mathcal{P}}^{S}\right]}{\text { Gauge }}
$$

where 'Gauge' means internal $\mathrm{SU}(2)$ rotations that reduce to $\mathrm{U}(1)$ on $S$, diffeomorphisms preserving $S$, and the motions generated by the Hamiltonian constraint. The quotient by 
diffeomorphisms identifies any two Hilbert spaces associated with sets $\mathcal{P}$ that can be mapped into another by a diffeomorphism on $S$. Thus, what matters is only the spins labelling punctures, not the locations of individual punctures. Unfortunately, we do not have yet a complete control over the quantum Hamiltonian constraint, despite the recent progress on this front [17]. To proceed, we make a rather weak assumption about the quantum dynamics: namely, that generically there is at least one solution of this constraint in $\mathcal{H}_{P}^{V} \otimes \mathcal{H}_{\mathcal{P}}^{S}$ for any set $\mathcal{P}$ of punctures labelled by spins.

We are not interested in this full Hilbert space since it includes, e.g., states of gravitational waves far away from $\Delta$. Rather, we wish to consider only states of the horizon of a black hole with area $A$. Thus we trace over the 'volume' states to construct an density matrix $\rho_{\text {bh }}$ describing a maximal-entropy mixture of surface states for which the area of the horizon lies in the range $A_{S} \pm \ell_{P}^{2}$. The statistical mechanical black hole entropy is then given by $S_{\mathrm{bh}}=-\operatorname{Tr} \rho_{\mathrm{bh}} \ln \rho_{\mathrm{bh}}$. As usual, this can be computed simply by counting states: $S_{\mathrm{bh}}=\ln N_{\mathrm{bh}}$ where $N_{\mathrm{bh}}$ is the number of Chern-Simons surface states satisfying the area constraint.

Fortunately, the eigenvalues of the area operator are explicitly known. For the case now under consideration, they are given by 8 :

$$
8 \pi \gamma \ell_{P}^{2} \sum_{p} \sqrt{j_{p}\left(j_{p}+1\right)}
$$

where $j_{p}$ are the spins labelling the punctures. Using this and the fact (from Chern Simons theory) that for a large number of punctures the dimension of $\mathcal{H}_{\mathcal{P}}^{S}$ grows as

$$
\operatorname{dim} \mathcal{H}_{\mathcal{P}}^{S} \sim \prod_{j_{p} \in \mathcal{P}}\left(2 j_{p}+1\right)
$$

it is straightforward to calculate the entropy. For large $A$ it is given by

$$
S_{\mathrm{bh}}=\frac{\gamma_{0}}{4 \ell_{P}^{2} \gamma} A_{S}, \quad \gamma_{0}=\frac{\ln 2}{\pi \sqrt{3}},
$$

where the appearance of $\gamma$ can be traced back directly to the formula for the eigenvalues of the area operator, (7). Thus, in the limit of large area, the entropy is proportional to the area of the horizon. If we set $\gamma=\gamma_{0}$, the statistical mechanical entropy is given precisely by the Bekenstein-Hawking formula.

Are there independent checks on this preferred value? The answer is in the affirmative. One can carry out this calculation for Reissner-Nordstrom as well as dilatonic black holes. A priori it could have happened that, to obtain the Bekenstein-Hawking value, one would have to re-adjust the Immirzi parameter for each value of the electric or dilatonic charge. This does not happen. The entropy is still given by (9) and hence by the Bekenstein-Hawking value when $\gamma=\gamma_{0}$.

To summarize, we first introduced a black hole sector of the gravitational phase space and then quantized it using the by now well-developed framework of non-perturbative quantum gravity together with results from Chern-Simons theory. We found that the entropy is proportional to the area irrespective of the value of the Immirzi parameter $\gamma$ and that a single choice of $\gamma$ yields the Bekenstein-Hawking coefficient irrespective of the parameters labeling the non-rotating black hole. 
We conclude with a few remarks:

i) One can show [16] that different values of $\gamma$ correspond to different 'sectors', that is, unitarily inequivalent representations of the canonical commutation relations. The spectrum of the area operator is different in each representation. As usual in such situations, the 'correct' sector can only be singled out by additional input (see, for example, the analogous ambiguity in the loop quantization of Maxwell theory [18]). The Bekenstein-Hawking calculation can be regarded as serving this purpose. However, the full significance of $\gamma$ is yet to be understood.

ii) A detailed calculation shows that the states which dominate the counting correspond to punctures all of which have labels $j=1 / 2$. Thus, there is a curious similarity between our detailed results and John Wheeler's "It from Bit" picture [20] of the origin of black hole entropy.

iii) So far, we have only considered non-rotating black holes. However, the basic ideas underlying this framework apply also to the rotating case.

iv) Our approach provides only an 'effective' description of a quantum black hole, for we first isolated a black hole sector classically and then quantized that sector. The issue of extracting this sector from a complete theory of quantum gravity is yet to be explored. Nonetheless, it is rather striking that subtle results from quite different areas - classical general relativity, quantum geometry and Chern-Simons theory - fit tightly without a mismatch to provide a coherent picture of the microstates of a black hole. The detailed implications of this picture for the black hole evaporation process are now being explored [19].

Acknowledgements: We are grateful to Don Marolf and Carlo Rovelli for their comments. This work was supported in part by the NSF grant PHY95-14240 and by the Eberly research funds of Penn State. AA, JB and KK acknowledge support from the Erwin Schrödinger Institute for Mathematical Sciences. AC was supported by DGAPA of UNAM. 


\section{REFERENCES}

[1] S. Carlip, Class. Quant. Grav. 12, 2853 (1995).

[2] G. Horowitz, "Quantum states of black holes", hep-th/9704072.

[3] C. Rovelli, Helv. Phys. Acta. 69, 583 (1996).

[4] A. Ashtekar, Phys. Rev. Lett. 57, 2244 (1986); Lectures on Non-perturbative Canonical Gravity, (World Scientific, Singapore, 1991).

[5] C. Rovelli and L. Smolin, Nucl. Phys. B331, 80 (1990).

[6] A. Ashtekar and J. Lewandowski, in Knots and Quantum Gravity, edited by J. Baez (Oxford U.P., Oxford, 1994); J. Baez, Lett. Math. Phys. 31, 213 (1994);

[7] C. Rovelli and L. Smolin, Phys. Rev. D52, 5743 (1995); J. Baez, Adv. Math. 117, 253 (1996).

[8] C. Rovelli and L. Smolin, Nucl. Phys. B442, 593 (1995); S. Fritelli, L. Lehner, C. Rovelli, Class. Quant. Grav. 13, 2921 (1996); K. Krasnov, "On the constant that fixes the area spectrum in canonical quantum gravity", gr-qc/9709058.

[9] A. Ashtekar, J. Lewandowski, Class. Quant. Grav. 14, 55 (1997).

[10] L. Smolin, J. Math. Phys. 36, 6417 (1995).

[11] C. Rovelli Phys. Rev. Lett. 77, 3288 (1996);

[12] K. Krasnov, Phys. Rev. D55, 3505 (1997).

[13] K. Krasnov, "On statistical mechanics of a Schwarzschild black hole", Gen. Rel. Grav. (in press), gr-qc/9605047.

[14] J. F. Plebanski, J. Math. Phys. 18, 2511 (1977); J. Samuel, Pramana J. Phys. 28, L429 (1987); T. Jacobson and L. Smolin, Phys. Lett. 196, 39 (1987).

[15] F. Barbero, Phys. Rev. D54, 1492 (1996);

[16] G. Immirzi, "Quantum gravity and Regge calculus", gr-qc/9701052; C. Rovelli, T. Thiemann, "The Immirzi parameter in quantum general relativity", gr-qc/9705059.

[17] T. Thiemann, Phys. Lett. B380 (1996), 257-264.

[18] A. Corichi and K. Krasnov, "Loop quantization of Maxwell theory and electric charge quantization", hep-th/9703177.

[19] K. Krasnov, "Quantum geometry and thermal radiation from black holes", preprint CGPG-97/9-4.

[20] J. Wheeler, in Sakharov Memorial Lectures on Physics, vol. 2, edited by L. Keldysh and V. Feinberg (Nova Science, New York, 1992). 